\title{
New Developments in Peptide Receptor Radionuclide Therapy
}

\author{
Guillaume P. Nicolas ${ }^{1}$, Alfred Morgenstern ${ }^{2}$, Margret Schottelius ${ }^{3}$, and Melpomeni Fani ${ }^{4}$ \\ ${ }^{I}$ Division of Nuclear Medicine, University Hospital Basel, University of Basel, Basel, Switzerland; ${ }^{2}$ Joint Research Centre, \\ Directorate for Nuclear Safety and Security, European Commission, Karlsruhe, Germany; ${ }^{3}$ Institute of Pharmaceutical \\ Radiochemistry, Technische Universität München, Garching, Germany; and ${ }^{4}$ Division of Radiopharmaceutical Chemistry, University \\ Hospital Basel, University of Basel, Basel, Switzerland
}

Peptide receptor radionuclide therapy (PRRT) is an established treatment for nonoperable or metastatic neuroendocrine neoplasms that highly and frequently express somatostatin receptors. More generally, PRRT is an attractive therapy option for delivering cytotoxic radiation to tumor cells through specific binding of a radiolabeled peptide to a molecular target. The development of imaging companions gave rise to the concept of radiotheranostics, important for in vivo tumor detection, characterization, and staging but also, and more importantly, for individual patient selection and treatment. The success of somatostatin receptor targeting paved the way for the clinical translation of other peptide-based radiopharmaceuticals targeting, for example, the receptors cholecystokinin 2, gastrin-releasing peptide, neurokinin-1, and C-X-C motif chemokine 4. Although historically the Auger emitter ${ }^{111}$ In and the high-energy $\beta^{-}$emitter ${ }^{90} Y$ were used, most PRRT are currently performed with the medium-energy $\beta^{-}$emitter ${ }^{177} \mathrm{Lu}$, whereas $a$ emitters are increasingly studied in various clinical applications.

Key Words: targeted radionuclide therapy; theranostics; G-protein coupled receptors; beta radiation therapy; alpha radiation therapy

J Nucl Med 2019; 60:167-171

DOI: 10.2967/jnumed.118.213496

$\mathbf{P}$ eptide receptor radionuclide therapy (PRRT) is part of the wider concept of targeted radionuclide therapy. PRRT delivers destructive radiation to cancer cells via radiolabeled peptides able to bind specifically to peptide receptors expressed in higher density on the tumor cell membrane than in nontumor tissues. This is the case for many G-protein-coupled receptors, whose overexpression is linked to numerous human malignancies. Regulatory peptides targeting G-protein-coupled receptors are a rich source of vectors (1) that can be chemically tuned to transport radioactivity while preserving their receptor affinity. PRRT is used in the treatment of metastatic or unresectable cancers through systemic or, more occasionally, locoregional administration.

Three types of radiation are used in PRRT: $\beta^{-}$particles (electrons), $\alpha$ particles, and Auger electrons (2), with a strong focus on $\beta^{-}$emitters (e.g., ${ }^{177} \mathrm{Lu}$ and ${ }^{90} \mathrm{Y}$ ). Because $\beta$ particles have a long range in tissues $(0.05-12 \mathrm{~mm})$, neighboring cells around the

Received Nov. 5, 2018; revision accepted Dec. 14, 2018.

For correspondence or reprints contact: Melpomeni Fani, Division of Radiopharmaceutical Chemistry, University Hospital Basel, University of Basel, Petersgraben 4, 4031 Basel, Switzerland.

E-mail: melpomeni.fani@usb.ch

Published online Dec. 20, 2018.

COPYRIGHT (C 2019 by the Society of Nuclear Medicine and Molecular Imaging. targeted cell are also irradiated (cross-fire effect). This effect is considered ideal for targeting large tumors with a heterogeneous target distribution. In contrast, $\alpha$ particles (e.g., ${ }^{213} \mathrm{Bi}$ and ${ }^{225} \mathrm{Ac}$ ) have a very short range in tissues $(20-100 \mu \mathrm{m})$, irradiating volumes with cellular dimensions and therefore sparing normal surrounding tissues from cytotoxic radiation. Their linear energy transfer is much higher than the one of $\beta^{-}$particles (50-230 vs. $0.2 \mathrm{keV} / \mu \mathrm{m})$, making $\alpha$ radiation far more cytotoxic. Finally, Auger emitters (e.g., ${ }^{111} \mathrm{In}$ ), having a very short range in tissue $(<20 \mu \mathrm{m}$, subcellular dimensions) and intermediate linear energy

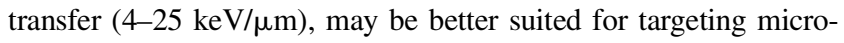
scopic disease (i.e., micrometastases), such as in the adjuvant setting. All above-mentioned radionuclides are radiometals (Table 1).

Peptide-based radiotherapeutics require a chelator that stably chelates the radiometal in vivo (3), in order to exclude deposition of free radiometal in normal tissues. The conjugation of a universal chelator, such as DOTA, allows radiolabeling with different radiometals sharing similar chemical properties, such as the trivalent radiometals ${ }^{90} \mathrm{Y},{ }^{177} \mathrm{Lu},{ }^{225} \mathrm{Ac}$, or ${ }^{68} \mathrm{Ga}$ (used in PET). This versatility enabled the development of radiotheranostics, which are essential for selecting patients who are suitable for PRRT. Such an approach may provide more efficient and personalized patient care, because properties of the vector, type of radionuclide, and dose of radioactivity can be tailored to address specific clinical needs.

Radiolabeled somatostatin analogs have been archetypal for the development of PRRT, since neuroendocrine neoplasms (NENs) strongly and frequently overexpress somatostatin receptor (sstr). This article discusses new approaches to overcoming current limitations, improving treatment outcomeconsidering that the objective response is still limited and few patients can be cured-and extending the application of PRRT beyond NENs. Also, recent developments in PRRT targeting the receptors cholecystokinin 2, gastrin-releasing peptide (GRPR), neurokinin-1, and C-X-C motif chemokine 4 (CXCR4) are presented. In addition, targeting of the prostate-specific membrane antigen (PSMA) with radiolabeled PSMA inhibitors has been recently a major breakthrough in targeted radionuclide therapy of prostate cancer. Strictly speaking, PSMA ligands are not peptides but peptidomimetics and PSMA is not a receptor but a type II transmembrane protein; therefore, PSMA-targeted radionuclide therapy is not discussed here.

\section{SOMATOSTATIN IN NEN AND BEYOND}

More than $20 \mathrm{y}$ after the pioneering work in isolated centers in Europe such as Rotterdam, Basel, and Milan, PRRT with radiolabeled sstr agonists (e.g., DOTATOC or DOTATATE, Table 2) is part of the standard of care for NENs (4). Recently, the 
TABLE 1

Radionuclides Used for PRRT in Patients

\begin{tabular}{|c|c|c|c|c|c|}
\hline Isotope & $\begin{array}{c}\text { Main decay } \\
\text { mode }\end{array}$ & Half-life & Therapeutic particle and energy & Tissue penetration depth & Status \\
\hline${ }^{177} \mathrm{Lu}$ & $\beta^{-}, y$ & $6.7 \mathrm{~d}$ & $\beta^{-}, E_{(\text {mean })}=0.149 \mathrm{MeV}, E_{(\max )}=0.497 \mathrm{MeV}$ & Mean, 0.5 mm; maximum, 2 mm & MA \\
\hline $90 Y$ & $\beta^{-}$ & $2.7 \mathrm{~d}$ & $\beta^{-}, E_{(\text {mean })}=0.937 \mathrm{MeV}, E_{(\max )}=2.284 \mathrm{MeV}$ & Mean, $2.5 \mathrm{~mm}$; maximum, $11 \mathrm{~mm}$ & MA \\
\hline${ }^{111} \ln$ & $\mathrm{EC}$ & $2.8 \mathrm{~d}$ & Auger $\mathrm{e}^{-}, 0.13-25.6 \mathrm{keV}$ & $0.25 \mathrm{~nm}-13.6 \mu \mathrm{m}$ & $\mathrm{MA}$ \\
\hline${ }^{213} \mathrm{Bi}$ & a & $45.6 \min$ & $\mathrm{a}, 5.9 / 8.4 \mathrm{MeV}$ & $85 \mu \mathrm{m}$ & CS \\
\hline${ }^{225} \mathrm{Ac}$ & $a$ & $9.9 \mathrm{~d}$ & $a, 5.8-8.4 \mathrm{MeV}$ & 47-85 $\mu \mathrm{m}$ & CS \\
\hline${ }^{212} \mathrm{~Pb}$ & $a, \beta^{-}, E C$ & $10.6 \mathrm{~h}$ & a, 6.2-8.9 Mev & 50-100 $\mu \mathrm{m}$ & CS \\
\hline
\end{tabular}

randomized phase III study NETTER-1 showed an improved objective response, an improved quality of life, improved progressionfree survival, and a clear trend toward an overall survival benefit from PRRT with 4 cycles of ${ }^{177} \mathrm{Lu}$-DOTATATE (plus long-actingrelease single-dose octreotide) compared with long-acting-release double-dose octreotide alone (5,6). Consequently, ${ }^{177} \mathrm{Lu}-\mathrm{DOTA}-$ TATE ( ${ }^{177} \mathrm{Lu}$-oxodotreotide) received marketing authorization for patients with metastatic and progressive midgut NEN. It is expected that approval of ${ }^{177} \mathrm{Lu}$-DOTATOC $\left({ }^{177} \mathrm{Lu}\right.$-edotreotide) will follow completion of the COMPETE-NCT03049189 phase III trial comparing ${ }^{177} \mathrm{Lu}$-DOTATOC with a mammalian target of rapamycin inhibitor, everolimus, in patients with inoperable, progressive gastroenteropancreatic NEN. These and other trials (e.g., OCCLURANDOM-NCT02230176) should more precisely determine the position of PRRT in the current clinical algorithm with regard to other systemic therapies, such as everolimus and sunitinib.

Routes other than intravenous administration may be interesting for enhancing the therapeutic and safety window of PRRT. NENs and liver metastases are often highly perfused, and the intraarterial route can exploit the first-pass effect to more efficiently treat liverdominant disease. Such an approach can also be used for inoperable primary tumors to downstage the disease in the neoadjuvant setting $(7,8)$. However, large comparative prospective trials, to support its wider use, are lacking.

\section{New Somatostatin Analogs}

An important evolutionary step is the use of radiolabeled sstr antagonists instead of agonists. Several clinical and preclinical studies (9) suggest significant improvement in the diagnostic sensitivity and therapeutic efficacy of the antagonists. ${ }^{177} \mathrm{Lu}$-satoreotide tetraxetan $\left({ }^{177} \mathrm{Lu}\right.$-OPS201 or ${ }^{177} \mathrm{Lu}-\mathrm{DOTA}-J \mathrm{R} 11$ [Ipsen], Table 2) shows higher tumor accumulation and absorbed dose (Fig. 1) and higher numbers of DNA double-strand breaks than ${ }^{177} \mathrm{Lu}$-DOTATATE (10-12). These characteristics are partly due to the higher number of available binding sites and the longer tumor residence time for the antagonist. ${ }^{177} \mathrm{Lu}$-OPS201 is currently under

TABLE 2

Peptide Analogs Used for PRRT in Patients

\begin{tabular}{|c|c|c|}
\hline Compound & Chemical formula & Status \\
\hline DOTATATE & DOTA-D-Phe-cyclo(Cys-Tyr-D-Trp-Lys-Thr-Cys)Thr & MA \\
\hline DOTATOC & DOTA-D-Phe-cyclo(Cys-Tyr-D-Trp-Lys-Thr-Cys)Thr(ol) & ODD \\
\hline $\begin{array}{l}\text { OPS201 (DOTA-JR11); OPS202 } \\
\quad \text { (NODAGA-JR11) }\end{array}$ & $\begin{array}{l}\text { (DOTA-/NODAGA-)Cpa-cyclo[D-Cys-Aph(Hor)-D-Aph(Cbm)-Lys-Thr- } \\
\text { Cys]-D-Tyr-NH2 }\end{array}$ & CS \\
\hline CP04 & DOTA-(D-Glu) $)_{6}$-Ala-Tyr-Gly-Trp-Met-Asp-Phe- $\mathrm{NH}_{2}$ & CS \\
\hline DOTA-PP-F11N & DOTA-(D-Glu) $)_{6}$-Ala-Tyr-Gly-Trp-Nle-Asp-Phe- $\mathrm{NH}_{2}$ & CS \\
\hline $\mathrm{RM}^{*}$ & $\begin{array}{l}\text { DOTA-4-amino-1-carboxymethyl-piperidine-D-Phe-GIn-Trp-Ala-Val-Gly- } \\
\text { His-Sta-Leu- } \mathrm{NH}_{2}\end{array}$ & CS \\
\hline NeoBOMB1* & $\begin{array}{l}\text { DOTA- } p \text {-aminomethylaniline-diglycolic acid-D-Phe-GIn-Trp-Ala-Val-Gly- } \\
\text { His- } \mathrm{NH}-\mathrm{CH}\left[\left(\mathrm{CH}_{2}-\mathrm{CH}\left(\mathrm{CH}_{3}\right)_{2}\right]_{2}\right.\end{array}$ & CS \\
\hline $\begin{array}{l}\left.\text { DOTA-/DOTAGA-[Thi }{ }^{8}, \operatorname{Met}\left(\mathrm{O}_{2}\right)^{11}\right]- \\
\text { substance } \mathrm{P}\end{array}$ & DOTA-/DOTAGA-Arg-Pro-Lys-Pro-Gln-Gln-Phe-Thi-Gly-Leu-Met $\left(\mathrm{O}_{2}\right)$ & CS \\
\hline Pentixather & Cyclo(D-3-iodo-Tyr-(NMe)-D-Orn(AMBS-DOTA)-Arg-2-Nal-Gly) & CS \\
\hline \multicolumn{3}{|c|}{$\begin{array}{l}\text { *No clinical data available on therapeutic radiotracers. } \\
\mathrm{Cpa}=4 \text {-Cl-phenylalanine; Aph(Hor) }=4 \text {-amino-L-hydroorotyl-phenylalanine; D-Aph(Cbm) }=\mathrm{D}-4 \text {-amino-carbamoyl-phenylalanine } \\
\text { MBS }=4 \text {-aminomethyl benzoic acid. } \\
\text { MA = marketing authorization; ODD = orphan drug designation; CS = clinical studies. } \\
\text { Chemical structures for these compounds can be found in Supplemental Table } 1 \text { (available at http://jnm.snmjournals.org). }\end{array}$} \\
\hline
\end{tabular}




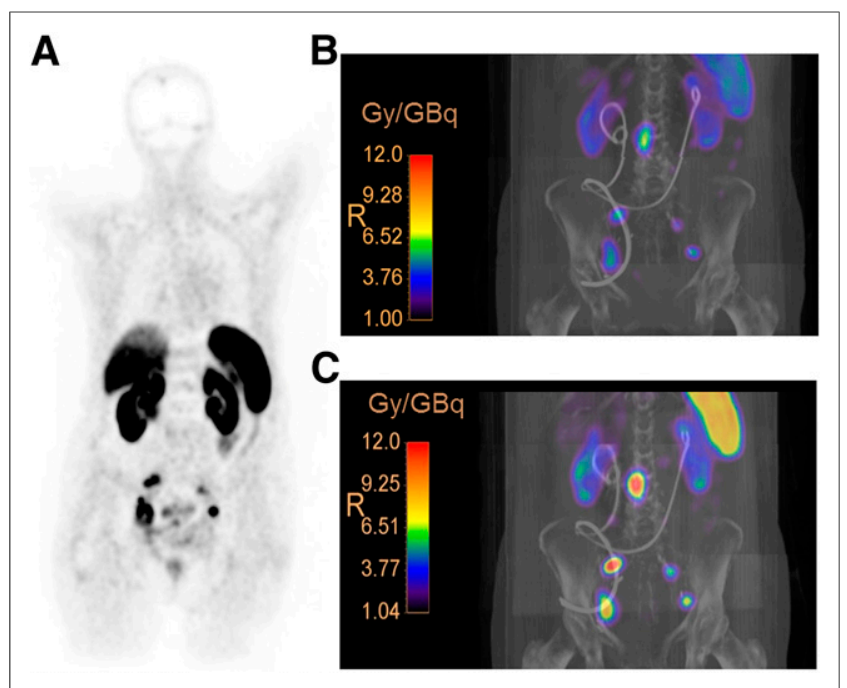

FIGURE 1. Patient with neuroendocrine neoplasia (G3) of bladder with lymph node metastases. (A) ${ }^{68} \mathrm{Ga}$-DOTATATE PET scan demonstrates progression with pelvic lymph node metastases after surgery. ( $\mathrm{B}$ and C) Three-dimensional voxel dosimetry after administration of $\sim 1 \mathrm{GBq}$

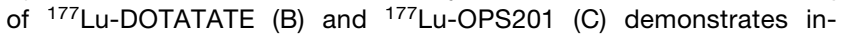
creased ( 4 times) tumor dose of ${ }^{177}$ Lu-OPS201, compared with ${ }^{177}$ Lu-DOTATATE, implying potential for greater therapeutic efficacy. (Courtesy of Prof. Damian Wild; patient was included in previously reported pilot study (10).)

evaluation in a multicenter phase I/II study (NCT02592707) and in a single-center theranostic study (NCT02609737). Also, ${ }^{68} \mathrm{Ga}-$ satoreotide trizoxetan $\left({ }^{68} \mathrm{Ga}-\mathrm{OPS} 202\right.$ or ${ }^{68} \mathrm{Ga}-\mathrm{NODAGA}-J R 11$ [Ipsen], Table 2), showing improved affinity for sstr compared with ${ }^{68} \mathrm{Ga}$-OPS201, demonstrated substantially higher sensitivity than the agonist ${ }^{68} \mathrm{Ga}$-DOTATOC in a prospective phase I/II study involving 12 gastroenteropancreatic neuroendocrine tumor patients (13)

By introducing the albumin-binding moiety Evans blue, ${ }^{177} \mathrm{Lu}-$ DOTA-EB-TATE was developed as a long-circulating sstr agonist (14). In 8 patients, its prolonged blood circulation resulted in a 7.9-fold increase in tumor dose, compared with ${ }^{177} \mathrm{Lu}$-DOTATATE, but at the cost of an even greater increase in renal and bone marrow absorbed doses, questioning its potential advantage over ${ }^{177}$ Lu-DOTATATE.

Use of multivalent or heterovalent vectors to simultaneously target several receptors concomitantly expressed in the same cancer cell is an interesting approach to overcoming tumor heterogeneity, resistance, and change in phenotype during disease progression (15).

\section{New Indications (Non-NEN)}

The larger number of binding sites recognized by sstr antagonists may open new horizons for sstr-targeted PRRT. In some nonneuroendocrine neoplasias, the level of binding of antagonists reaches the level of agonists (e.g., ${ }^{177}$ Lu-DOTATATE) in well-differentiated NEN (16). Indications such as metastatic breast and small cell lung cancers are currently investigated in second line in a basket multicenter phase I/II trial with the theranostic pair ${ }^{68} \mathrm{Ga}-\mathrm{OPS} 202 /{ }^{177} \mathrm{Lu}-\mathrm{OPS} 201$ (NCT03773133).

Beyond oncologic indications, initial reports suggest a potential role for sstr-targeted PRRT in inflammatory conditions, such as sarcoidosis and atherosclerosis $(17,18)$. In a feasibility study involving only 2 patients with refractory multiorgan involvement of sarcoidosis, ${ }^{177} \mathrm{Lu}$-DOTATOC showed treatment effects (17), whereas a retrospective analysis of a limited number of oncology patients indicated that ${ }^{177} \mathrm{Lu}$-DOTATATE reduces atherosclerotic plaque activity (18).

\section{a-Therapy}

${ }^{213} \mathrm{Bi}$-DOTATOC was investigated in 25 metastatic NEN patients progressing after ${ }^{90} \mathrm{Y}-/{ }^{177} \mathrm{Lu}$-DOTATOC therapy (activities ranging from 2.6 to $21 \mathrm{GBq}$ in $1-5$ cycles). An interim report on the first 7 patients (8) showed moderate renal and hematologic toxicity, but long-term follow-up data are still pending. ${ }^{213} \mathrm{Bi}$ DOTATOC treatments resulted in a high proportion of long-lasting antitumor responses, including one complete remission, even with large-volume disease (Fig. 2). However, the current supply limitations for high-activity ${ }^{225} \mathrm{Ac} /{ }^{213} \mathrm{Bi}$ generators prevent larger confirmatory prospective studies and have instead motivated the use of ${ }^{225} \mathrm{Ac}$ or ${ }^{212} \mathrm{~Pb}$, which are $\beta^{-}$emitters but act as an in vivo generator for the $\alpha$ emitter ${ }^{212} \mathrm{Bi}$. ${ }^{212} \mathrm{~Pb}$-DOTAMTATE (AlphaMedix; RadioMedix, Inc.) has just entered a phase I study (NCT03466216), and ${ }^{225}$ Ac-DOTATOC has been administered to 40 patients with progressive NEN (19). The maximum tolerated dose of ${ }^{225}$ Ac-DOTATOC was established at $40 \mathrm{MBq}$ in a single fraction and $25 \mathrm{MBq}$ in 2 fractions at a 4-mo interval (19). Larger prospective comparative studies are needed to identify the most suitable patients for ${ }^{225}$ Ac-DOTATOC therapy. Importantly, translocation of radioactive daughter nuclides from the chelator should be considered a potential safety hazard for $\alpha$ emitters with multiple $\alpha$-emitting daughters.

\section{Combination Therapy}

Combined use of various radionuclides (e.g., ${ }^{90} \mathrm{Y}$ and ${ }^{177} \mathrm{Lu}$ ), sequentially or concomitantly, might optimize radiation delivery across the entire spectrum of lesion size that is often present in one individual. This approach has shown some potential for increasing survival in comparison to the single-radionuclide approach (20).

Integration of PRRT into multimodality-therapy protocols might improve treatment response (21). The use of radiosensitizing chemotherapeutics in combination with ${ }^{90} \mathrm{Y}-$ and ${ }^{177} \mathrm{Lu}-\mathrm{DOTA}-$ TATE has shown additive effects resulting in an unprecedented objective response (22). This may particularly be interesting in bulky, higher-grade, or ${ }^{18} \mathrm{~F}-\mathrm{FDG}$-avid NENs, in which the potential risk of long-term hematologic toxicity is balanced by the more aggressive course of the disease. Prospective clinical trials

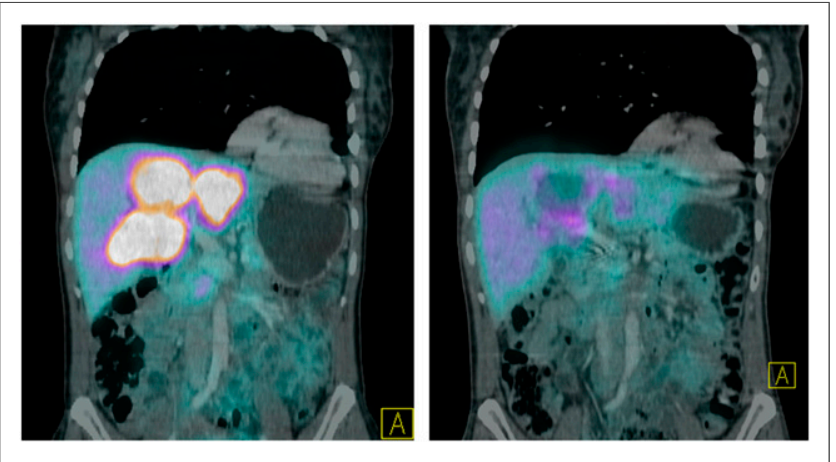

FIGURE 2. ${ }^{68} \mathrm{Ga}-\mathrm{DOTATOC}$ PET/CT images before (left) and after (right) intraarterial therapy with $14 \mathrm{GBq}$ of ${ }^{213} \mathrm{Bi}$-DOTATOC, demonstrating response of multiple liver lesions. (Reprinted from (8).) 
combining PRRT and chemotherapy are under way (NCT02736448, NCT02358356) with agents such as 5-fluorouracil, capecitabine, and temozolomide.

Molecularly targeted therapeutics may be important to attain synergy with PRRT. For instance, concurrent inhibition of the DNA repair mechanisms with a poly-[ADP-ribose]-polymerase 1 inhibitor results in increased DNA double-strand breaks (23).

\section{GASTRIN IN MEDULLARY THYROID CANCER}

Virtually all medullary thyroid cancers express the cholecystokinin 2 receptor, for which the endogenous ligand is gastrin. PRRT with radiolabeled gastrin analogs is therefore an attractive treatment option for patients with recurrent or metastatic medullary thyroid cancer. Of large libraries, the analogs ${ }^{177} \mathrm{Lu}-\mathrm{PP}-\mathrm{F} 11 \mathrm{~N}$ (Table 2) and ${ }^{111} \mathrm{In}-\mathrm{CP} 04$ (PP-F11, Table 2), as a surrogate for ${ }^{177} \mathrm{Lu}-$ or ${ }^{90}$ Y-labeled CP04, are currently in early prospective trials (LUMED/ NCT02088645 and GRAN-T-MTC/NCT03246659, respectively) to determine safety, maximum tolerated dose, biodistribution, and dosimetry $(24,25)$. Preliminary clinical data identified the stomach as a potential dose-limiting organ, whereas the renal absorbed dose was rather low.

Promising perspectives involve the use of adjuvant protease inhibitors to prevent enzymatic tracer degradation in vivo and improve the tumor uptake and pharmacokinetics of enzymatically vulnerable radiolabeled gastrin analogs (26).

\section{BOMBESIN IN PROSTATE AND BREAST CANCER}

GRPR is overexpressed in, among other malignancies, prostate and breast cancers. The ${ }^{68} \mathrm{Ga}$-labeled GRPR antagonists RM2 and NeoBOMB1 (Table 2) are currently under clinical evaluation in prostate cancer and gastrointestinal stromal tumor. Treatment in humans has not been reported yet but has been evaluated in animal models $(27,28)$. Interestingly, various ongoing studies compare ${ }^{68} \mathrm{Ga}$-GRPR antagonists with ${ }^{68} \mathrm{Ga}$-PSMA analogs (29) (NCT03604757, NCT03606837, NCT03698370), which is essential to understand the role of each radiotracer in the management of prostate cancer patients. The fact that GRPR and PSMA seem to be expressed at different stages of the disease, and that the biodistribution of the radiotracers is fundamentally different, may result in 2 complementary rather than competitive theranostic approaches.

Radiolabeled GRPR antagonists may also have a potential in estrogen receptor-positive breast tumors, which represent most breast cancer patients (30).

\section{SUBSTANCE P IN GLIOMA}

PRRT of gliomas has been investigated with the substance P analog DOTA-/DOTAGA-SP (Table 2) (31-34), targeting the neurokinin-1 receptor that is overexpressed in World Health Organization grade II-IV gliomas. To overcome the blood-brain barrier and to improve tumor uptake, administration is conducted locoregionally via an implanted catheter connected to a subcutaneous port.

Initial results with ${ }^{90} \mathrm{Y}$ and ${ }^{177} \mathrm{Lu}$ indicated clinical potential $(31,32)$; however, recent studies focused on the $\alpha$ emitters ${ }^{213} \mathrm{Bi}$ and ${ }^{225} \mathrm{Ac}$, allowing more selective tumor cell irradiation and limiting toxicity to adjacent healthy brain tissue. ${ }^{213} \mathrm{Bi}$-DOTASP has been examined in 61 patients with grade II-IV gliomas (up to a cumulative activity of $14.1 \mathrm{GBq})(33,34)$. A subgroup analysis in 7 patients with secondary glioblastoma showed a median overall survival of 18.6 mo after conversion to grade IV (33). In another subgroup of 20 patients with recurrent glioblastoma, a median overall survival of 23.6 mo was found, compared with 14.6 mo after standard therapy alone (34). Treatment with the longer-lived ${ }^{225} \mathrm{Ac}$ has been initiated and 20 glioma patients have been enrolled in a dose escalation study (from 10 to $42 \mathrm{MBq}$ ) investigating the intratumoral or intercavitary injection of ${ }^{225} \mathrm{Ac}-$ DOTAGA-SP (34). The analysis of therapeutic efficacy and patient recruitment are ongoing.

\section{CXCR4 IN CANCER}

CXCR4 is an attractive target for theranostic interventions since it is overexpressed in hematologic malignancies, such as multiple myeloma, leukemia, and non-Hodgkin lymphoma, and in some solid cancers (e.g., lung cancer, adrenocortical cancer, and highgrade NEN). Many high-affinity ligands targeting CXCR4 have been developed, among which the theranostic pair ${ }^{68} \mathrm{Ga}$-pentixafor $/{ }^{177} \mathrm{Lu} /{ }^{90}$ Y-pentixather is the most advanced one (Table 2) (35). Preclinically, efficient eradication of CXCR4-positive human leukemic blasts was shown in a patient-derived xenograft model of acute lymphocytic leukemia (36). In a pilot study, 8 multiple myeloma patients (37) were treated with ${ }^{177} \mathrm{Lu}$ - or ${ }^{90} \mathrm{Y}$-labeled pentixather (7.6-23.5 GBq or 2.6-6.3 GBq, respectively) and showed a high initial response rate (37).

Given the fact that hematologic cancers are strongly disseminated diseases, ${ }^{177} \mathrm{Lu}$ might not be the optimal radionuclide, and current efforts are directed toward implementing $\alpha$ therapy using ${ }^{213} \mathrm{Bi}-/ 225 \mathrm{Ac}$-pentixather. Thorough evaluation of hematologic safety is indispensable because of the physiologic expression of CXCR4 in hematopoietic stem cells.

\section{FUTURE PERSPECTIVES}

Despite the great advances that PRRT represents in the management of NEN, ${ }^{177} \mathrm{Lu}$-oxodotreotide (also known as ${ }^{177} \mathrm{Lu}$-DOTATATE and Lutathera [Advanced Accelerator Applications]) is currently the only approved peptide-based radiotherapeutic agent. More effort is needed to expand PRRT beyond NENs, which involves the development of new radiotheranostics and the validation of new targets. Additionally, tumor-specific upregulation of targets by pharmacologic or epigenetic interventions, as well as the use of heterovalent ligands, may be valuable options to enhance tumor uptake and overcome resistance. Furthermore, the use of alternative theranostic pairs of radionuclides, such as radioisotopes of scandium $\left({ }^{43 / 44 / 47} \mathrm{Sc}\right)$ and terbium $\left({ }^{149 / 152 / 155 / 161} \mathrm{~Tb}\right)(38)$, might open novel theranostic applications. To further optimize the potential of various radionuclides and to promote synergistic combination therapies, a deeper understanding of all relevant radiobiologic processes is required, including DNA-repair mechanisms and ontarget and off-target (bystander and abscopal) effects (39). Finally, more precise image-based dosimetry, to establish dose-effect relationships, and development of biomarkers, such as multitranscript gene blood assays (40), are expected to improve the prediction of outcome after PRRT.

\section{DISCLOSURE}

No potential conflict of interest relevant to this article was reported. 


\section{REFERENCES}

1. Vaudry H, Tonon MC, Vaudry D. Editorial: trends in regulatory peptides. Front Endocrinol (Lausanne). 2018;9:125.

2. Dash A, Knapp FF, Pillai MR. Targeted radionuclide therapy: an overview. Curr Radiopharm. 2013;6:152-180.

3. Price EW, Orvig C. Matching chelators to radiometals for radiopharmaceuticals. Chem Soc Rev. 2014;43:260-290.

4. Hicks RJ, Kwekkeboom DJ, Krenning E, et al. ENETS consensus guidelines for the standards of care in neuroendocrine neoplasia: peptide receptor radionuclide therapy with radiolabeled somatostatin analogues. Neuroendocrinology. 2017;105: 295-309.

5. Strosberg J, El-Haddad G, Wolin E, et al. Phase 3 trial of ${ }^{177} \mathrm{Lu}$-dotatate for midgut neuroendocrine tumors. N Engl J Med. 2017;376:125-135.

6. Strosberg J, Wolin E, Chasen B, et al. Health-related quality of life in patients with progressive midgut neuroendocrine tumors treated with ${ }^{177} \mathrm{Lu}$-dotatate in the phase III NETTER-1 trial. J Clin Oncol. 2018;36:2578-2584.

7. Partelli S, Bertani E, Bartolomei M, et al. Peptide receptor radionuclide therapy as neoadjuvant therapy for resectable or potentially resectable pancreatic neuroendocrine neoplasms. Surgery. 2018;163:761-767.

8. Kratochwil C, Giesel FL, Bruchertseifer F, et al. ${ }^{213}$ Bi-DOTATOC receptor-targeted alpha-radionuclide therapy induces remission in neuroendocrine tumours refractory to beta radiation: a first-in-human experience. Eur J Nucl Med Mol Imaging. 2014;41:2106-2119.

9. Fani M, Nicolas GP, Wild D. Somatostatin receptor antagonists for imaging and therapy. J Nucl Med. 2017;58(suppl):61S-66S.

10. Wild D, Fani M, Fischer R, et al. Comparison of somatostatin receptor agonist and antagonist for peptide receptor radionuclide therapy: a pilot study. $\mathrm{J} \mathrm{Nucl}$ Med. 2014;55:1248-1252.

11. Nicolas GP, Mansi R, McDougall L, et al. Biodistribution, pharmacokinetics, and dosimetry of ${ }^{177} \mathrm{Lu}-,{ }^{90} \mathrm{Y}-$, and ${ }^{111} \mathrm{In}$-labeled somatostatin receptor antagonist OPS201 in comparison to the agonist ${ }^{177} \mathrm{Lu}$-DOTATATE: the mass effect. $\mathrm{J}$ Nucl Med. 2017;58:1435-1441.

12. Dalm SU, Nonnekens J, Doeswijk GN, et al. Comparison of the therapeutic response to treatment with a ${ }^{177} \mathrm{Lu}$-labeled somatostatin receptor agonist and antagonist in preclinical models. J Nucl Med. 2016;57:260-265.

13. Nicolas GP, Schreiter N, Kaul F, et al. Sensitivity comparison of ${ }^{68}$ Ga-OPS202 and ${ }^{68} \mathrm{Ga}$-DOTATOC PET/CT in patients with gastroenteropancreatic neuroendocrine tumors: a prospective phase II imaging study. J Nucl Med. 2018;59:915921.

14. Zhang J, Wang H, Jacobson Weiss O, et al. Safety, pharmacokinetics and dosimetry of a long-acting radiolabeled somatostatin analogue ${ }^{177} \mathrm{Lu}-\mathrm{DOTA}-\mathrm{EB}-$ TATE in patients with advanced metastatic neuroendocrine tumors. $\mathrm{J} \mathrm{Nucl} \mathrm{Med}$. 2018;59:1699-1705.

15. Reubi JC, Maecke HR. Approaches to multireceptor targeting: hybrid radioligands, radioligand cocktails, and sequential radioligand applications. $J$ Nucl Med. 2017;58(suppl):10S-16S.

16. Reubi JC, Waser B, Macke H, Rivier J. Highly increased ${ }^{125}$ I-JR11 antagonist binding in vitro reveals novel indications for sst2 targeting in human cancers. J Nucl Med. 2017;58:300-306.

17. Lapa C, Kircher M, Hanscheid H, et al. Peptide receptor radionuclide therapy as a new tool in treatment-refractory sarcoidosis: initial experience in two patients. Theranostics. 2018;8:644-649.

18. Schatka I, Wollenweber T, Haense C, Brunz F, Gratz KF, Bengel FM. Peptide receptor-targeted radionuclide therapy alters inflammation in atherosclerotic plaques. J Am Coll Cardiol. 2013;62:2344-2345.

19. Kratochwil C, Bruchertseifer F, Giesel F, Apostolidis C, Haberkorn U, Morgenstern A. Ac-225-DOTATOC: an empiric dose finding for alpha particle emitter based radionuclide therapy of neuroendocrine tumors [abstract]. $\mathrm{J} \mathrm{Nucl}$ Med. 2015;56(suppl):1232.

20. Villard L, Romer A, Marincek N, et al. Cohort study of somatostatin-based radiopeptide therapy with [ ${ }^{90} \mathrm{Y}$-DOTA]-TOC versus [ $\left.{ }^{90} \mathrm{Y}-\mathrm{DOTA}\right]-\mathrm{TOC}$ plus $\left[{ }^{177} \mathrm{Lu}-\mathrm{DOTA}\right]-\mathrm{TOC}$ in neuroendocrine cancers. J Clin Oncol. 2012;30:11001106.
21. Gill MR, Falzone N, Du Y, Vallis KA. Targeted radionuclide therapy in combined-modality regimens. Lancet Oncol. 2017;18:e414-e423.

22. Kong G, Callahan J, Hofman MS, et al. High clinical and morphologic response using ${ }^{90}$ Y-DOTA-octreotate sequenced with ${ }^{177} \mathrm{Lu}$-DOTA-octreotate induction peptide receptor chemoradionuclide therapy (PRCRT) for bulky neuroendocrine tumours. Eur J Nucl Med Mol Imaging. 2017;44:476-489.

23. Nonnekens J, van Kranenburg M, Beerens CE, et al. Potentiation of peptide receptor radionuclide therapy by the PARP inhibitor olaparib. Theranostics. 2016;6: 1821-1832.

24. Sauter AW, Mansi R, Hassiepen U, et al. Targeting of the cholecystokinin-2 receptor with the minigastrin analog ${ }^{177}$ Lu-DOTA-PP-F11N: does the use of protease inhibitors further improve in vivo distribution? J Nucl Med. July 12, 2018 [Epub ahead of print].

25. Maina T, Konijnenberg MW, KolencPeitl P, et al. Preclinical pharmacokinetics, biodistribution, radiation dosimetry and toxicity studies required for regulatory approval of a phase I clinical trial with ${ }^{111} \mathrm{In}$-CP04 in medullary thyroid carcinoma patients. Eur J Pharm Sci. 2016;91:236-242.

26. Kaloudi A, Nock BA, Lymperis E, et al. Impact of clinically tested NEP/ACE inhibitors on tumor uptake of [ ${ }^{111}$ In-DOTA]MG11: first estimates for clinical translation. EJNMMI Res. 2016;6:15.

27. Dumont RA, Tamma M, Braun F, et al. Targeted radiotherapy of prostate cancer with a gastrin-releasing peptide receptor antagonist is effective as monotherapy and in combination with rapamycin. $J$ Nucl Med. 2013;54:762-769.

28. Dalm SU, Bakker IL, de Blois E, et al. ${ }^{68} \mathrm{Ga} /{ }^{177} \mathrm{Lu}-\mathrm{NeoBOMB} 1$, a novel radiolabeled GRPR antagonist for theranostic use in oncology. J Nucl Med. 2017;58: 293-299.

29. Minamimoto R, Hancock S, Schneider B, et al. Pilot comparison of ${ }^{68} \mathrm{Ga}-\mathrm{RM} 2$ PET and ${ }^{68} \mathrm{Ga}$-PSMA-11 PET in patients with biochemically recurrent prostate cancer. J Nucl Med. 2016;57:557-562.

30. Stoykow C, Erbes T, Maecke HR, et al. Gastrin-releasing peptide receptor imaging in breast cancer using the receptor antagonist ${ }^{68} \mathrm{Ga}-\mathrm{RM} 2$ and PET. Theranostics. 2016;6:1641-1650.

31. Cordier D, Krolicki L, Morgenstern A, Merlo A. Targeted radiolabeled compounds in glioma therapy. Semin Nucl Med. 2016;46:243-249.

32. Cordier D, Forrer F, Kneifel S, et al. Neoadjuvant targeting of glioblastoma multiforme with radiolabeled DOTAGA-substance P: results from a phase I study. J Neurooncol. 2010;100:129-136.

33. Krolicki L, Bruchertseifer F, Kunikowska J, et al. Prolonged survival in secondary glioblastoma following local injection of targeted alpha therapy with ${ }^{213} \mathrm{Bi}$ substance P analogue. Eur J Nucl Med Mol Imaging. 2018;45:1636-1644.

34. Krolicki L, Bruchertseifer F, Kunikowska J, et al. Targeted alpha therapy of glioblastoma multiforme: clinical experience with ${ }^{213} \mathrm{Bi}$ - and ${ }^{225} \mathrm{Ac}$-substance P. In: 10th Symposium on Targeted Alpha Therapy. Brussels, Belgium: European Commission, Joint Research Centre; 2017:24.

35. Schottelius M, Osl T, Poschenrieder A, et al. $\left[{ }^{177} \mathrm{Lu}\right]$ pentixather: comprehensive preclinical characterization of a first CXCR4-directed endoradiotherapeutic agent. Theranostics. 2017;7:2350-2362.

36. Habringer S, Lapa C, Herhaus $P$, et al. Dual targeting of acute leukemia and supporting niche by CXCR4-directed theranostics. Theranostics. 2018;8:369383.

37. Lapa C, Herrmann K, Schirbel A, et al. CXCR4-directed endoradiotherapy induces high response rates in extramedullary relapsed multiple myeloma. Theranostics. 2017;7:1589-1597.

38. Müller C, Domnanich KA, Umbricht CA, van der Meulen NP. Scandium and terbium radionuclides for radiotheranostics: current state of development towards clinical application. Br J Radiol. 2018;91:20180074.

39. Pouget JP, Georgakilas AG, Ravanat JL. Targeted and off-target (bystander and abscopal) effects of radiation therapy: redox mechanisms and risk/benefit analysis. Antioxid Redox Signal. 2018;29:1447-1487.

40. Bodei L, Kidd M, Modlin IM, et al. Measurement of circulating transcripts and gene cluster analysis predicts and defines therapeutic efficacy of peptide receptor radionuclide therapy (PRRT) in neuroendocrine tumors. Eur J Nucl Med Mol Imaging. 2016;43:839-851. 